\title{
Characterization of the Liquid Crystal Display Modulation. Optimization for Some Applications
}

J. Campos ${ }^{a, *}$, A. Márquez ${ }^{a, \dagger}$, J. Nicolás ${ }^{a}$, I. Moreno ${ }^{b}$, C. IEMmi ${ }^{c}$, J.A. DaVIS ${ }^{d}$ AND M..J. YZUEL ${ }^{a}$

${ }^{a}$ Dept. Física, Universitat Autonoma de Barcelona 08193 - Bellaterra (Barcelona), Spain

${ }^{b}$ Dpt. Ciencia y Tecnología de Materiales, Universidad Miguel Hernández Elche (Alicante), Spain

${ }^{c}$ Fac. de Ciencias Experimentales y Naturales, Universidad de Buenos Aires Buenos Aires, Argentina

${ }^{d}$ Dept. of Physics, San Diego State University, San Diego, USA

In this paper we revise recent results of our team in the optimization of twisted nematic liquid crystal displays to be used as spatial light modulators for image processing and diffractive optics. In general two kind of responses are desired for the mentioned applications: amplitude-only and phase-only modulations. However, it is not a trivial task to find the polarization configurations for which these responses are obtained. We show that a reverse-engineering approach is needed to optimize the liquid crystal display response. According to this reverse-engineering approach the modulation characteristics can be calibrated by evaluating the modulation response in a few polarization configurations. These results are used to fit the liquid crystal display behavior to a simplified physical model, which uses two modulation parameters. We demonstrate that the degree of accuracy of this model is very high, thus enabling the prediction of the modulation behavior of the display at other polarization configurations. Therefore, we can perform computer searches for the optimum orientation of the polarizing elements to obtain the required optical transmission. We demonstrate the need to use short wavelengths and the need to insert wave plates in front and behind the liquid crystal displays to obtain either amplitude-only or phase-only regime.

PACS numbers: $42.79 . \mathrm{Kr}, 42.79 . \mathrm{Hp}, 42.25 . \mathrm{Ja}$

*corresponding author; e-mail: Juan.Campos@uab.es

†Present address: Dept. Física, Ingeniería de Sistemas y Teoría de la Seńal, Universidad de Alicante, Spain 


\section{Introduction}

Among the different liquid crystal display technologies the twisted nematic liquid crystal display (TN-LCD) is the most extended device [1]. TN-LCDs extracted from video projectors or pocket televisions have been widely used as spatial light modulators (SLM) in applications such as image processing or diffractive optics. In optical signal processing they serve to introduce the scene and the filter in real time optical correlators [2-4]. TN-LCDs make also possible the implementation of diffractive optical elements (DOE) whose characteristics can be changed dynamically [5-8]. TN-LCDs are also interesting for wave front control in adaptive optics [9].

In general two kinds of optical responses are desired for these applications: amplitude-only modulation and phase-only modulation. Some strategies have been proposed to have the TN-LCD working in either the amplitude-only or the phase-only mode $[10,11]$. However, with new TN-LCDs these strategies lose effectiveness. New TN-LCDs have a larger resolution, forcing a decrease in the thickness of the device to provide a faster response for the addressing of the pixels. The decrease in the thickness shortens the dynamic modulation range of the device. Davis et al. [12] demonstrated that the use of the eigenvectors of the LCD [13] results in a great improvement in phase-only modulation with respect to the case in which only linearly polarized light is considered.

We demonstrated in Ref. [14] an optimization procedure leading to amplitude-only and phase-only modulations for thin-LCDs. The eigenvectors of the LCD can be considered as a particular case of our more general procedure. We have to apply a novel elliptically polarized light approach and we have to use a short wavelength. This optimization procedure is based on a simplified model we proposed for the physical properties of the TN-LCD [15]. This model is able to predict with a high degree of accuracy the amplitude and phase modulations introduced by the TN-LCD. The model assumes two modulation parameters which vary with the applied voltage. The values for these two voltage dependent parameters can be found by means of a reverse-engineering approach given in Ref. [15].

In this paper we revise the procedure proposed to obtain a required complex amplitude modulation with TN-LCDs. First, in Sec. 2 we show the approach that we follow to characterize the performance of the TN-LCD according to the model that we proposed in Ref. [15]. Then, in Sec. 3 we demonstrate the optimization procedure leading to amplitude-only or phase-only modulations, which are the interesting regimes for a number of applications. Finally, in Sec. 4 we give the conclusions of the work.

\section{Reverse-engineering approach with prediction capability}

\subsection{Model for the performance of the $T N-L C D$}

TN-LCDs are filled with nematic liquid crystal compounds. They are composed of rodlike molecules arranged with their long axes pointing in a common 
LCD LCD

input face output face

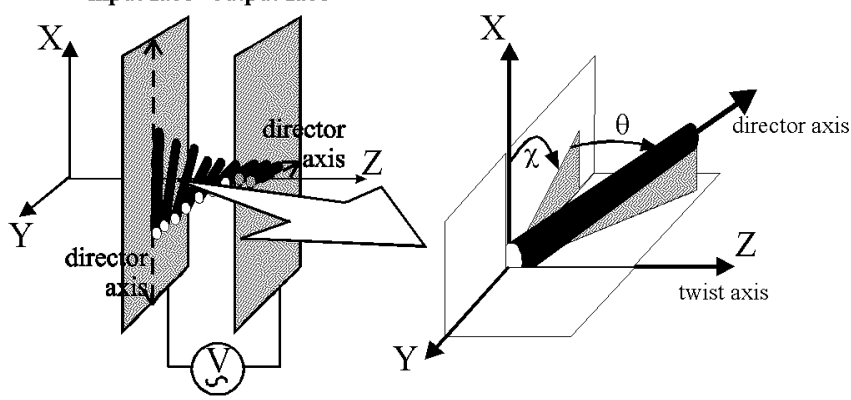

Fig. 1. Twist $\chi$ and tilt $\theta$ angles of the molecular director $n$.

orientation, the so-called director axis orientation $n$. Nematic materials exhibit uniaxial optical and electrical properties. Under the application of an electric field the director axis reorientates. The polarization state of light traversing the cell depends on the optic axis orientation, which has changed with the field-induced director axis reorientation [16]. In Fig. 1 we show the distribution of the director axis $n$ in a TN-LCD. To determine the orientation of the director axis we need two angles: the twist angle $\chi$ and the tilt angle $\theta$. The director axis twists with respect to the so-called twist axis, that we consider parallel to the $Z$-axis. The total twist angle $\alpha$ from the entrance to the output face is usually about 90 degrees in twisted-nematic devices. When a voltage is applied the molecules tend to orientate parallel to the $Z$-axis. We note that the liquid crystal molecules are forced to lay parallel to the surfaces $(\theta=0)$ at both sides of the TN-LCD.

In order to analyze and study the optical performance of the liquid crystal devices we need to know the orientation of the molecular director $n$ across the device. In Fig. 2a and $b$ we show the realistic profiles as a function of the applied voltage respectively for the twist $\chi$ and the tilt $\theta$ angles. We can see that these profiles

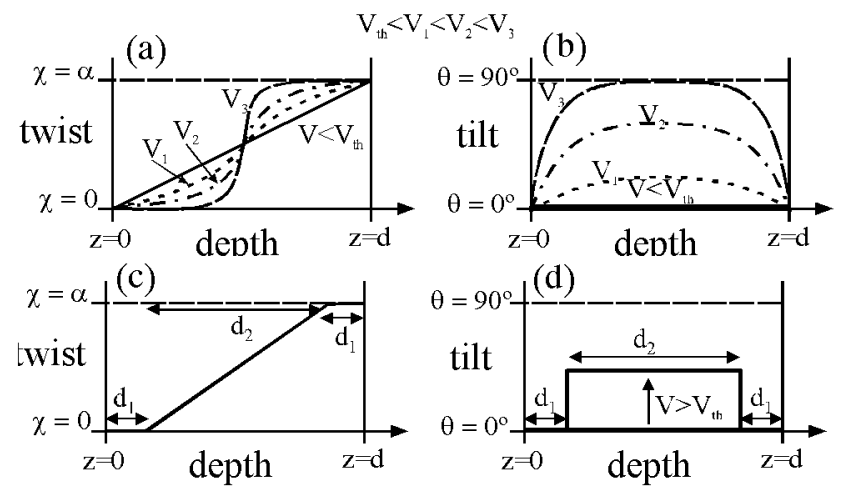

Fig. 2. Behavior for the twist $\chi$ and the tilt $\theta$ angles for different voltages: (a) and (b) - realistic profiles, (c) and (d) - the proposed model. 
vary nonlinearly with the depth $z$ of the device. These nonlinearities are produced because the molecular director in the vicinity of the surfaces of the TN-LCD is not free to move as the director in the center [16]. To calculate the director profile across the device we need the values for the physical constants of the liquid crystal material and the fabrication parameters of the TN-LCD, which are not provided by the manufacturers. Thus, we are forced to use a reverse-engineering approach.

In Ref. [15] we proposed a simplified model for the TN-LCD, based on the models by Lu and Saleh [11] and by Coy et al. [17]. The profiles for the twist $\chi$ and the tilt $\theta$ angles according to our proposal are shown in Fig. $2 \mathrm{c}$ and d, respectively. We divide the TN-LCD in three regions, as illustrated in Fig. 3. On the one hand, two edge layers, with a voltage dependent thickness $d_{1}(V)$ each of them, where the liquid crystal molecules are unable to tilt and twist, thus acting as two wave plates. On the other hand, a central part, with a thickness $d_{2}(V)$ which exhibits a homogeneous tilt and a linear twist with the depth of the device. Therefore, the total thickness of the TN-LCD $d$ is $d=2 d_{1}(V)+d_{2}(V)$. In principle $d_{1}=0$, i.e. no edge effect exists, when the applied voltage is smaller than the threshold voltage $V_{\mathrm{th}}$.

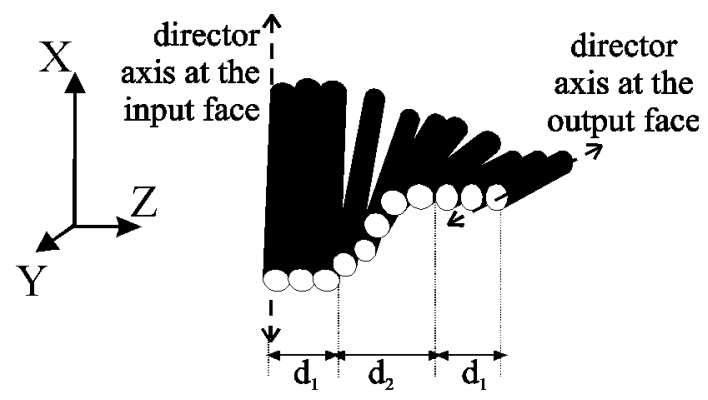

Fig. 3. Diagram of the proposed model for the TN-LCD, with the two edge layers of thickness $d_{1}$ and the central part of thickness $d_{2}$.

As a result of the model we define two modulation parameters, the so-called birefringence $\beta$ of the central part and the birefringence $\delta$ of each edge layer, whose values vary with the applied voltage. The expressions for these birefringence parameters are given by $\beta(V)=\pi d_{2}(V) \Delta n(V) / \lambda$ and $\delta(V)=\pi d_{1}(V) \Delta n_{\max } / \lambda$, where $\lambda$ is the wavelength of the incident light. $\Delta n$ is the difference between the extraordinary and the ordinary indices of refraction along the propagation direction of the light in the TN-cell. $\Delta n$ varies with the tilt angle $\theta$, which changes with the applied voltage $V$. It is a maximum, $\Delta n_{\max }$, when $\theta=0$, as at the edge layers. Hence, the total birefringence of the TN-cell is $\delta+\beta+\delta$. We note that the retardance generated between the extraordinary and the ordinary rays propagating parallel to the $Z$-axis across the TN-LCD is twice this value, i.e. the total retardance is $2 \delta+2 \beta+2 \delta$.

The Jones matrix $M_{\mathrm{LCD}}(\alpha, \beta, \delta)$ of the device according to this model is given by $[14,15]$ 


$$
M_{\mathrm{LCD}}(\alpha, \beta, \delta)=\exp (-\mathrm{i}(\beta+2 \delta)) R(-\alpha)\left(\begin{array}{cc}
X-\mathrm{i} Y & Z \\
-Z & X+\mathrm{i} Y
\end{array}\right)
$$

where

$$
\begin{aligned}
& X=\cos \gamma \cos 2 \delta-\frac{\beta}{\gamma} \sin \gamma \sin 2 \delta, \quad Y=\cos \gamma \sin 2 \delta+\frac{\beta}{\gamma} \sin \gamma \cos 2 \delta \\
& Z=\frac{\alpha}{\gamma} \sin \gamma
\end{aligned}
$$

with $\gamma=\left(\alpha^{2}+\beta^{2}\right)^{1 / 2}$, and $R(\theta)$ is the two-dimensional rotation matrix, i.e.,

$$
R(\theta)=\left(\begin{array}{cc}
\cos \theta & \sin \theta \\
-\sin \theta & \cos \theta
\end{array}\right) \text {. }
$$

We can see that the Jones matrix of the device depends on the total twist angle $\alpha$, and on the two birefringence parameters $\beta$ and $\delta$ which vary with the applied voltage.

\subsection{Characterization results}

In the reverse-engineering approach, there are a number of parameters that we have to calculate to fully characterize the performance of the TN-LCD. We can group these unknowns in two different categories: the voltage independent and the voltage dependent unknowns. There are two voltage independent unknowns: the orientation $\Psi_{\mathrm{D}}$ of the molecular director at the input face of the device (with respect to the laboratory coordinate system), and the total twist angle $\alpha$. The two voltage dependent unknowns are the birefringences $\beta(V)$ and $\delta(V)$ according to the model we propose. The work in this paper has been done with a LCD panel Sony Model LCX012BL, extracted from a video projector Sony VPL-V500. The Sony LCX012BL is a $3.3 \mathrm{~cm}$ diagonal active matrix thin film transistor (TFT) panel, with VGA resolution $(640 \times 480$ pixels $)$. The pixels are square with a pixel center to center separation of $41 \mu \mathrm{m}$ and a clear aperture width of $34 \mu \mathrm{m}$. We use the electronics of the video projector to send the voltage to the pixels of the LCD.

The voltage independent parameters have been calculated by means of known techniques [18-20]. For these measurements we use the unexpanded beams for four different wavelengths: $633 \mathrm{~nm}$ from a He-Ne laser and the 514, 488, and $458 \mathrm{~nm}$ from an $\mathrm{Ar}^{+}$laser. We illuminate the TN-LCD with linearly polarized light. In Table we summarize the values obtained for the voltage independent unknowns. We can see that with these techniques we also obtain the value for the maximum birefringence $\beta_{\max }$ exhibited by the TN-LCD, which corresponds to the birefringence value when there is no voltage applied to the device. We have found that the birefringence values $\beta_{\max }$ at different wavelengths are scaled. Our experimental ratios are $1.3,1.4$, and 1.56 , respectively, for the 514,488 , and $458 \mathrm{~nm}$ wavelengths compared with the $633 \mathrm{~nm}$ wavelength.

The voltage dependent parameters $\beta(V)$ and $\delta(V)$ are calculated using the technique we propose in Ref. [15]. We insert the TN-LCD between two linear polarizers with their transmission axes in specific orientations. The normalized 
TABLE

Values of the voltage independent parameters. $\Psi_{\mathrm{d}}$ the orientation angle of the director axis at the entrance of LCD.

\begin{tabular}{c|c|c|c|c|c}
\hline \hline$\alpha$ & $\Psi_{\mathrm{d}}$ & \multicolumn{4}{|c}{$\beta_{\max }(\mathrm{deg})$} \\
\cline { 3 - 6 }$(\mathrm{deg})$ & $(\mathrm{deg})$ & $633 \mathrm{~nm}$ & $514 \mathrm{~nm}$ & $488 \mathrm{~nm}$ & $458 \mathrm{~nm}$ \\
\hline-92 & 46 & 147 & 193 & 208 & 231
\end{tabular}

transmission of the TN-LCD inserted between two polarizers, with transmission axes at angles $\varphi_{1}$ and $\varphi_{2}$ with respect to the director at the input and at the output faces of the device respectively, is given by

$$
\left.T\left(\varphi_{1}, \varphi_{2}\right)\right)=\left[X \cos \left(\varphi_{1}-\varphi_{2}\right)+Z \sin \left(\varphi_{1}-\phi_{2}\right)\right]^{2}+\left[Y \cos \left(\varphi_{1}+\varphi_{2}\right)\right]^{2} .
$$

The nonlinear curve fitting of intensity transmission measurements using Eq. (4) provide us with the values for $\beta(V)$ and $\delta(V)$. In order to obtain a better accuracy the measurements have been taken at three specific configurations of the polarizers $\left(\left(\varphi_{1}=0, \varphi_{2}=+90^{\circ}\right),\left(\varphi_{1}=+45^{\circ}, \varphi_{2}=-45^{\circ}\right),\left(\varphi_{1}=+22.5^{\circ}, \varphi_{2}=\right.\right.$ $\left.+112.5^{\circ}\right)$ ), using the four wavelengths $(633 \mathrm{~nm}, 514 \mathrm{~nm}, 488 \mathrm{~nm}$, and $458 \mathrm{~nm})$. In our fit we use the wavelength dependent ratios obtained for the off-state birefringence $\left(\beta_{\max }\right)$ to scale the values of $\beta(V)$ and $\delta(V)$ for the different wavelengths. All the experiments were conducted with the brightness and contrast controls of the video projector at 50 and 100, respectively. We have measured the intensity at intervals of 10 gray scale levels (GSL). For the Sony VPL-V500 video projector electronics, the voltage decreases monotonically when the gray level increases. In Fig. $4 \mathrm{a}$ and $\mathrm{b}$ we show the set of values obtained for the birefringences $\beta(V)$ and $\delta(V)$ at the four wavelengths. The last data point, labeled OFF, corresponds to the measurement taken with the video projector switched off.

In Fig. $4 \mathrm{a}$, the $\beta$ values increase monotonically with gray level. At GSL $=0$, $\beta$ is approximately zero. This means that the liquid crystal molecules in the central
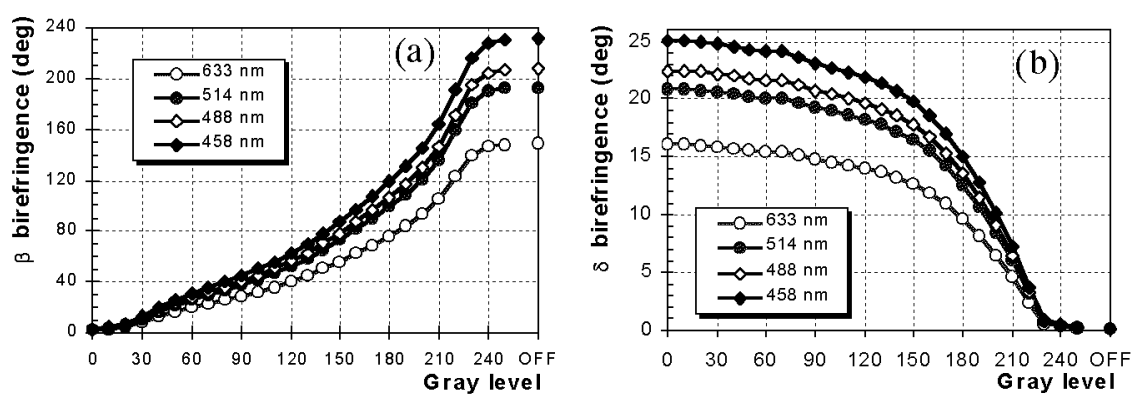

Fig. 4. Values obtained for the voltage dependent parameters at the four wavelengths. (a) $\beta(V)$, (b) $\delta(V)$. 
region of the LCD are totally tilted, and the applied voltage exceeds the saturation voltage. Above GSL $=230, \beta$ reaches the off-state value. Thus, the applied voltage is lower than the threshold voltage $V_{\text {th }}$. In Fig. $4 \mathrm{~b}$ at the off-state state point $\delta$ is nearly zero, i.e. no edge effect exists in the off-state. The $\delta$ birefringence increases with the increase in the applied voltages (decrease in the gray levels), achieving a maximum value $\delta_{\max }$ of approximately 25 degrees $(458 \mathrm{~nm}$ ).

\subsection{Accuracy of the proposed model}

The complete knowledge of $\beta(V)$ and $\delta(V)$ allows the calculation of the intensity transmission curves for any other configuration. To show this point let us consider an arbitrary configuration $\left(\varphi_{1}=0, \varphi_{2}=45^{\circ}\right)$. In Fig. 5 we show the experimental measurement (symbols) and the theoretical curve (lines) for the $458 \mathrm{~nm}$ wavelength. The three different lines show the theoretical curves calculated using the different reverse-engineering models.

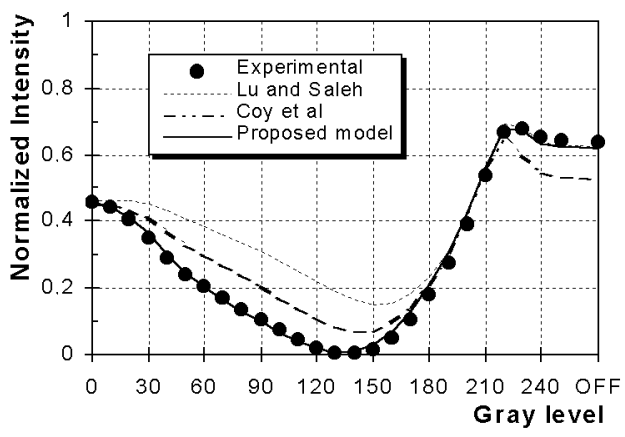

Fig. 5. Comparison between the experimental measurements at $458 \mathrm{~nm}$ for the configuration $\left(\varphi_{1}=0, \varphi_{2}=+45^{\circ}\right)$, and theoretical intensity transmission curves calculated with the three inverse models: Lu and Saleh, Coy et al., and the model we propose.

The Lu-Saleh model shows the worst agreement with the experimental data. In the case of the Coy et al. model, the best fit to the experimental data is obtained using the constant value $\delta=8.9$ degrees. The agreement is improved, but it is not good enough to provide accurate optical transmission calculations. Clearly the calculations made with the model we have proposed coincides perfectly with the experimental data. The excellent agreement validates the model and the characterization technique proposed to obtain the relations $\beta(V)$ and $\delta(V)$.

\section{Complex transmittance optimization}

\subsection{Elliptically polarized light setup}

In Ref. [14] we demonstrated a technique which allows us to obtain the required modulation with thin TN-LCDs. We generate an elliptical polarization 
state to illuminate the TN-LCD, and we detect another elliptical polarization state. The scheme for this architecture is illustrated in Fig. 6. The wave plates WP1 and WP2 introduce retardances of $2 \phi_{1}$ and $2 \phi_{2}$, respectively. The angles of the elements in front of the TN-LCD are referred to the orientation of the director at the input surface (which we suppose that coincides with the $X$-coordinate axis) while the angles of the elements behind the TN-LCD are measured with respect to the orientation of the director at the output surface.

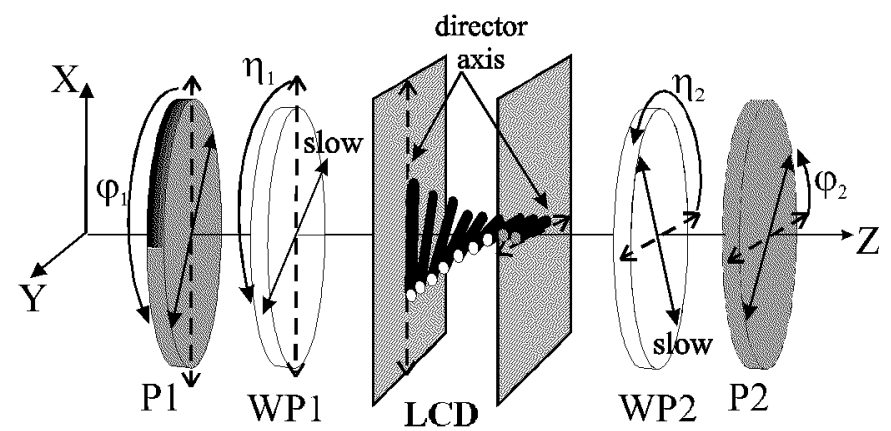

Fig. 6. Scheme for the generalized elliptical light setup.

The electric field $\boldsymbol{E}_{\text {OUT }}$ at the end of the optical setup, is given by

$$
\begin{aligned}
& \boldsymbol{E}_{\mathrm{OUT}}=P_{0} R\left(\alpha+\varphi_{2}\right) R\left(-\alpha-\eta_{2}\right) W_{0}\left(2 \phi_{2}\right) R\left(\alpha+\eta_{2}\right) \\
& \times M_{\mathrm{LCSLM}}(\alpha, \beta, \delta) R\left(-\eta_{1}\right) W_{0}\left(2 \phi_{1}\right) R\left(\eta_{1}\right)\left(\begin{array}{c}
\cos \varphi_{1} \\
\sin \varphi_{1}
\end{array}\right),
\end{aligned}
$$

where $P_{0}$ represents the Jones matrix of a linear polarizer oriented parallel to the $X$ coordinate axis, i.e.,

$$
P_{0}=\left(\begin{array}{ll}
1 & 0 \\
0 & 0
\end{array}\right)
$$

and $W(2 \phi)$ is the matrix of a wave plate centered on the coordinate axis, i.e.,

$$
W(2 \phi)=\left(\begin{array}{cc}
\exp (-\mathrm{i} \phi) & 0 \\
0 & \exp (+\mathrm{i} \phi)
\end{array}\right)
$$

Thus, the resultant complex amplitude $E_{t}$ transmitted by this system is given by

$$
E_{t}=\exp (-\mathrm{i}(\beta+2 \delta))\left(A_{\mathrm{RE}}+\mathrm{i} A_{\mathrm{IM}}\right),
$$

where $A_{\mathrm{RE}}$ and $A_{\mathrm{IM}}$ are real magnitudes whose expressions are as follows:

$$
\begin{aligned}
A_{\mathrm{RE}} & =X\left[\cos \phi_{1} \cos \phi_{2} \cos \left(\varphi_{1}-\varphi_{2}\right)-\sin \phi_{1} \sin \phi_{2} \cos \left(2\left(\eta_{1}-\eta_{2}\right)-\varphi_{1}+\varphi_{2}\right)\right] \\
& +Y\left[-\cos \phi_{1} \sin \phi_{2} \cos \left(2 \eta_{2}+\varphi_{1}-\varphi_{2}\right)-\sin \phi_{1} \cos \phi_{2} \cos \left(2 \eta_{1}-\varphi_{1}+\varphi_{2}\right)\right]
\end{aligned}
$$




$$
\begin{aligned}
& +Z\left[\cos \phi_{1} \cos \phi_{2} \sin \left(\varphi_{1}-\varphi_{2}\right)-\sin \phi_{1} \sin \phi_{2} \sin \left(2\left(\eta_{1}-\eta_{2}\right)-\varphi_{1}+\varphi_{2}\right)\right], \text { (9a) } \\
A_{\mathrm{IM}} & =X\left[-\cos \phi_{1} \sin \phi_{2} \cos \left(2 \eta_{2}-\varphi_{1}-\varphi_{2}\right)-\sin \phi_{1} \cos \phi_{2} \cos \left(2 \eta_{1}-\varphi_{1}-\varphi_{2}\right)\right] \\
& +Y\left[-\cos \phi_{1} \cos \phi_{2} \cos \left(\varphi_{1}+\varphi_{2}\right)+\sin \phi_{1} \sin \phi_{2} \cos \left(2\left(\eta_{1}+\eta_{2}\right)-\varphi_{1}-\varphi_{2}\right)\right] \\
& +Z\left[\cos \phi_{1} \sin \phi_{2} \sin \left(2 \eta_{2}-\varphi_{1}-\varphi_{2}\right)-\sin \phi_{1} \cos \phi_{2} \sin \left(2 \eta_{1}-\varphi_{1}-\varphi_{2}\right)\right] . \quad(9 \mathrm{~b})
\end{aligned}
$$

The intensity transmittance $T$ for this system is obtained as

$$
T=A_{\mathrm{RE}}^{2}+A_{\mathrm{IM}}^{2}
$$

and the phase-shift $\psi$ is given by

$$
\psi=-\beta-2 \delta+\operatorname{atan}\left(\frac{A_{\mathrm{IM}}}{A_{\mathrm{RE}}}\right) .
$$

This setup is general enough to include as particular cases the classical system with linearly polarized light and the eigenvectors system. We note that the classical system consisting of two linear polarizers, corresponds to the case $2 \phi_{1}=2 \phi_{2}=0$. The system to generate and detect the polarization eigenstates of the LCD [12] corresponds to the case $2 \phi_{1}=2 \phi_{2}=90^{\circ}$ with the restrictions $\eta_{1}=\eta_{2}=0$ and $\varphi_{2}=-\varphi_{1}$.

As $\beta(V)$ and $\delta(V)$ have already been measured, it is possible to perform a computer search for a desired modulation. In the optimization process we assign initial arbitrary values to the angles $\eta_{1}, \eta_{2}, \varphi_{1}$, and $\varphi_{2}$, thus, the transmittance $T$ and the phase-shift $\psi$ can be calculated using the analytical expressions in Eqs. (10) and (11). The optimization criterion to be applied depends on the complex transmittance modulation we are interested in.

In case two variable wave plates are available (for instance using SoleilBabinet compensators), the computation of the search for a desired modulation may also include $\phi_{1}$ and $\phi_{2}$ as free parameters. In any other case the available wave plates fix the values of $\phi_{1}$ and $\phi_{2}$.

For the computer search presented in the next sections, we have arbitrarily chosen two available wave plates with a retardance of $2 \phi_{1}=125$ degrees and $2 \phi_{2}=94.5$ degrees, respectively, for the wavelength $458 \mathrm{~nm}$. The rest of the magnitudes, $\eta_{1}, \eta_{2}, \varphi_{1}$, and $\varphi_{2}$, are left as free parameters in the optimization process. The case $2 \phi_{1}=2 \phi_{2}=0$ (only polarizers) has also been considered in a new computer search, corresponding to the situation when no wave plates are present. All the results that we show have been obtained with the $458 \mathrm{~nm}$ wavelength of an argon laser.

\subsection{Amplitude-only modulation}

In the amplitude-only modulation we seek a configuration that combines the next three features: flat phase response, high intensity contrast (ratio between the maximum $T_{\max }$ and the minimum $T_{\min }$ intensity transmission values), and monotonic and linear intensity transmission response with the gray level. The optimization criterion that we apply for the computer search is a trade off between these three conditions. 
Figure $7 \mathrm{a}$ and $\mathrm{b}$ shows respectively the intensity and the phase-shift values as a function of the gray level applied for the only polarizers configuration and for the general configuration with wave plates. We show the experimental values (symbols) and the predicted curves (lines). Excellent agreement between predicted and experimental values is obtained. For the case of only polarizers, the optimization search leads to the polarizer transmission axis angles $\varphi_{1}=91$, and $\varphi_{2}=89$ degrees. The transmission axes of both polarizers are practically perpendicularly oriented to the director axis at the input and at the output faces of the LCD, respectively. This is the configuration that has been usually considered in the literature as the optimum one for amplitude-only modulation. For the general case with wave plates the optimization search leads to the configuration given by the angles $\left(\varphi_{1}=116^{\circ}, \eta_{1}=96^{\circ}, \varphi_{2}=62^{\circ}, \eta_{2}=-7^{\circ}\right)$. We can see (Fig. 7 a) that in the general case with wave plates the intensity transmission is monotonic and with a very linear slope.
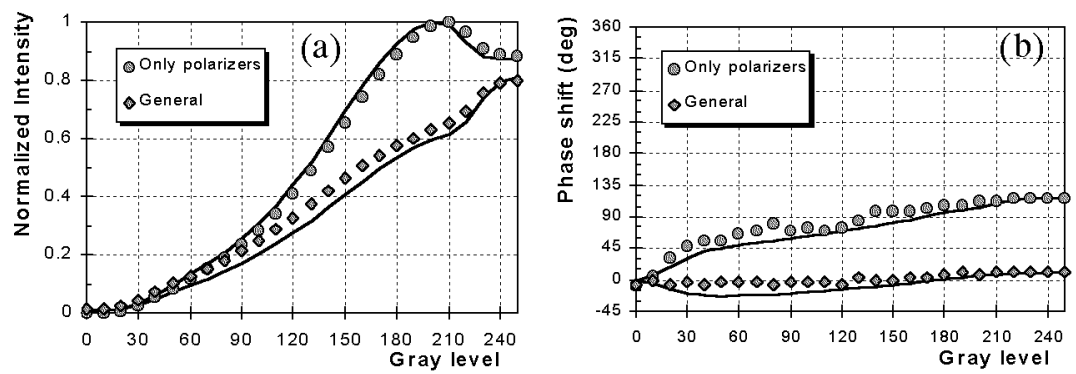

Fig. 7. Amplitude-only modulation. Only polarizers and general configurations. Normalized intensity (a), phase-shift (b). The symbols correspond to experimental measurements and the lines correspond to predicted values.

In display applications the main concern is to have a high contrast ratio, but in applications where amplitude-only modulation is required we also need a flat phase response. For the configuration with only polarizers the range of phase-shift is 124 degrees, which is far from a flat line. In the general case with wave plates, the amplitude-only modulation has been greatly improved with respect to the case with only polarizers: in the general case the range of phase-shift is as low as 18 degrees while keeping good values for the intensity contrast and a low value (about $1 \%$ ) for the minimum intensity transmission $T_{\min }$. An important result is that we have demonstrated that values as low as 18 degrees for the range of phase-shift are possible with a twisted-nematic device.

\subsection{Accurate prediction of phase-only modulation}

An optimum phase-only configuration must produce a flat amplitude modulation and a phase modulation with a phase depth of $2 \pi$ radians. The criterion 
we follow is to minimize the difference between the maximum and the minimum transmitted intensity $T_{\max }-T_{\min }$ over the entire voltage range. The intensity transmission may not be maximum, but we are interested in a configuration for which it remains constant.
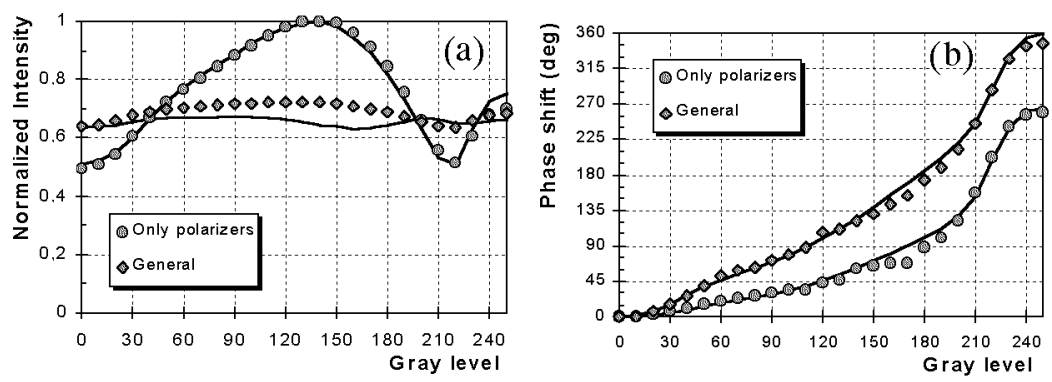

Fig. 8. Phase-only modulation. Only polarizers and general configurations. Normalized intensity (a), phase-shift (b). The symbols correspond to experimental measurements and the lines correspond to predicted values.

In Fig. 8a and b we show, respectively, the intensity and the phase-shift values versus the gray levels for the only polarizers configuration and for the general configuration with wave plates. We show the experimental values (symbols) and the predicted curves (lines). Once again we see the excellent agreement between predicted and experimental values. For the case of only polarizers, the optimization search leads to the polarizer transmission axis angles $\varphi_{1}=+22$, and $\varphi_{2}=-21$ degrees. For the general case with wave plates the optimization search leads to the angles $\left(\varphi_{1}=+26^{\circ}, \varphi_{2}=-16^{\circ}, \eta_{1}=0, \eta_{2}=+11^{\circ}\right)$.

As expected for a thin-LCD, the setup with only polarizers provides a very bad performance: $50 \%$ of intensity variation. For the general configuration with the wave plates we see that the optimum configuration provides an almost ideal phase-only modulation with a very uniform intensity transmission and a variation of the phase-shift $\Delta \psi$ of nearly 360 degrees. Therefore, the use of elliptically polarized light and short wavelengths allows us to obtain an almost ideal phase-only modulation configuration.

\section{Conclusions}

We have proposed an optimization procedure to obtain the desired complex amplitude transmission with a twisted-nematic LCD. We have obtained polarization configurations leading to a phase-only modulation and an amplitude-only modulation, which are interesting configurations in optical processing and in diffractive optics. We have demonstrated the need to use wave plates in front and behind the LCD and the need to illuminate with short wavelengths when working with thin-LCDs. The optimization procedure is based on a simple physical model which 
is able to predict with a high degree of accuracy the complex amplitude transmission of the TN-LCD. We use a reverse-engineering approach to characterize the two modulation parameters $\beta(V)$ and $\delta(V)$ in the model.

\section{Acknowledgments}

The work has been partially financed by Ministerio de Ciencia y Tecnología, project BFM2000-0036-C02-01 and by the Generalitat de Catalunya under project 1999SGR-96.

\section{References}

[1] V.G. Chigrinov, Liquid Crystal Devices: Physics and Applications, Artech House, Boston 1999.

[2] H.-K. Liu, J.A. Davis, R.A. Lilly, Opt. Lett. 10, 635 (1985).

[3] B. Javidi, F.T.S. Yu, Appl. Opt. 25, 2365 (1986).

[4] J. Campos, A. Márquez, M.J. Yzuel, J.A. Davis, D.M. Cottrell, I. Moreno, Appl. Opt. 39, 5965 (2000).

[5] E.C. Tam, Opt. Lett. 17, 369 (1992).

[6] V. Laude, Opt. Commun. 153, 134 (1998).

[7] J.A. Davis, J.C. Escalera, J. Campos, A. Márquez, M.J. Yzuel, C. Iemmi, Opt. Lett. 24, 628 (1999).

[8] A. Márquez, C. Iemmi, J.C. Escalera, J. Campos, S. Ledesma, J.A. Davis, M.J. Yzuel, Appl. Opt. 40, 2316 (2001).

[9] R. Dou, M.K. Giles, Opt. Lett. 20, 1583 (1995).

[10] N. Konforti, E. Marom, S.-T. Wu, Opt. Lett. 13, 251 (1988).

[11] K. Lu, B.E.A. Saleh, Opt. Eng. 29, 240 (1990).

[12] J.A. Davis, I. Moreno, P. Tsai, Appl. Opt. 37, 937 (1998).

[13] J.L. Pezzanitti, R.A. Chipman, Opt. Lett. 18, 1567 (1993).

[14] A. Márquez, C. Iemmi, I. Moreno, J.A. Davis, J. Campos, M.J. Yzuel, Opt. Eng. 40, 2558 (2001).

[15] A. Márquez, J. Campos, M.J. Yzuel, I. Moreno, J.A. Davis, C. Iemmi, A. Moreno, A. Robert, Opt. Eng. 39, 3301 (2000).

[16] S.-T. Wu, in: Spatial Light Modulator Technology: Materials, Devices and Applications, Ed. U. Efron, Marcel Dekker, New York 1995, ch. 1, p. 1.

[17] J.A. Coy, M. Zaldarriaga, D.F. Grosz, O.E. Martínez, Opt. Eng. 35, 15 (1996).

[18] C. Soutar, K. Lu, Opt. Eng. 33, 2704 (1994).

[19] J.A. Davis, D.B. Allison, K.G. D’Nelly, M.L. Wilson, I. Moreno, Opt. Eng. 38, 705 (1999).

[20] J.A. Davis, P. Tsai, K.G. D’Nelly, I. Moreno, Opt. Eng. 38, 929 (1999). 